\title{
An experimental analysis of the instability of nonaxisymmetric liquid bridges in a gravitational field
}

\author{
N. A. Bezdenejnykh, J. Meseguer, and J. M. Perales \\ IDRUPM, E.T.S.I. Aeronaticos, Universidad Politécnica de Madrid, 28040, Madrid, Spain
}

\begin{abstract}
The stability limits of nonaxisymmetric liquid bridges between equal in diameter. coaxial disks have been determined experimentally. Experiments have been performed by working with very small size liquid bridges. The experimental setup allows any orientation of the liquid bridge axis with respect to the local gravity vector acceleration. By appropriately orienting the liquid bridge axis, the influence on the stability limits of both the lateral and the axial component of the acceleration acting on the liquid bridge has been investigated.
\end{abstract}

\section{INTRODUCTION}

The fluid configuration considered consists of an isothermal liquid drop held by surface tension forces between two parallel, coaxial, solid disks of the same diameter, $2 R$, subjected to an acceleration field, as sketched in Fig. 1. The stability limits of this configuration (known as a liquid bridge) are determined by the following dimensionless parameters: the slendemess, $\Lambda$, defined as the ratio of the disks separation, $L$. to the disks common diameter, $\Lambda=L /(2 R)$, the dimensionless volume of the drop, defined as the ratio of the volume of the liquid bridge, $\mathrm{I}^{*}$, to the volume of a cylinder having the same length and diameter, $V$ $=V^{*} /\left(\pi R^{2} L\right)$, and the Bond number, $\mathbf{B}=\rho \mathrm{g} R^{2} / \sigma$, where $\rho$ is the difference between the density of the liquid and the density of the surrounding medium. $\mathbf{g}$ is the gravity acceleration (a vector which forms an angle $\varphi$ with the axis of the liquid bridge, as indicated in Fig. 1), and $\sigma$ is the surface tension. Note that since $\mathbf{g}$ is a vector, for the sake of simplicity, the Bond number has been also defined as a vector whose axial and lateral components $\left(B_{a}=|\mathbf{B}| \cos \varphi\right.$ and $B_{l}$ $=|\mathbf{B}| \sin \varphi$. respectively) correspond to the axial and the radial components of the acceleration vector.

As is well known, a liquid bridge can be stable or unstable depending on the relative values of the slenderness, the dimensionless volume, and the imposed perturbation. A given configuration can be represented by a point in a $\Lambda-V$ diagram (see Fig. 1). where two different regions can be identified: configurations lying inside the closed curve are stable whereas those lying outside are unstable. The border between both regions, which depends on the imposed perturbation, is the stability limit. The type of instability that leads to liquid bridge destabilization is different for different portions of the stability limit curve. For very small values of both the slendemess and the dimensionless volume (line $O-A$ ) the instability does not lead to a breakage but rather to a nonaxisymmetric detachment of the liquid bridge interface (the triple line) from the edges of the supporting disks. For larger values of the slenderness or of the volume (line $A-B$ ). a capillary instability leads to the breaking of the liquid column into two main drops whose relative volumes depend on both the value of the slenderness and on the magnitude of the imposed perturbation. This curve is known as minimum volume stability limit. Finally, the upper boundary of the stability region (line $O-B$ ) is due to a nonaxisymmetric instability and usually leads also to the spreading of the liquid over the lateral surfaces of the solid supports.

Equilibrium shapes and stability limits of capillary liquid bridges have been analyzed from both the theoretical and experimental points of view during the last decades, and many papers dealing with such fluid configurations have been published. ${ }^{1}$ However, most of the published papers deal only with axisymmetric equilibrium shapes (in which the direction of the acceleration is parallel to the liquid bridge axis, that is $\varphi=0, B_{l}=0$ ) and its stability (considering in some of them also nonaxisymmetric perturbations). Apart from the classical work of Coriell, Hardy, and Cordes, ${ }^{2}$ only recently have nonaxisymmetric equilibrium shapes been considered. An asymptotic analysis of the influence of lateral Bond number on the stability limit of liquid bridges having cylindrical volume $(Y=1)$ has been studied by Perales, ${ }^{3}$ and by Meseguer $e t a l^{4}$ who analyzed analytically and experimentally the influence of the combined effect of nonaxisymmetric perturbations (the lateral Bond number and noncoaxial supporting disks) on the stability limit. Recently, some numerical analyses dealing with the equilibrium shapes and stability limits of nonaxisymmetric liquid bridges have been published $^{5-9}$ and the same nonaxisymmetric configurations were used in an experimental work where the use of liquid bridges as accelerometers is explored. ${ }^{10}$

\section{APPARATUS}

There are several options for performing liquid bridge experiments on ground laboratories. Either small liquid bridges $s^{4,10.11}$ or the neutral buoyancy technique ${ }^{12}$ have mostly been used (although other techniques to avoid gravity effects have also been used for ground-based studies: ultrasound levitation, electric fields. and magnetic fields ${ }^{9,13}$ ). The first option has been chosen here. The apparatus used in the 


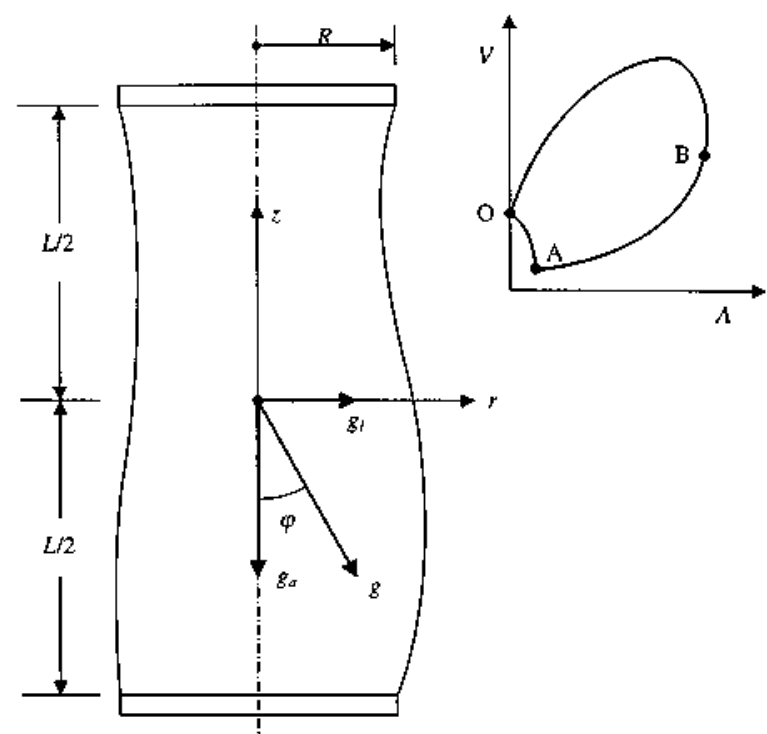

FIG. 1. Geometry, coordinate system, and sketch of a typical stability diagram of liquid bridges.

experiments consists of a millimetric liquid bridge cell (LBC) mounted on a platform, which can be oriented at any prescribed direction with respect to the local gravity direction (Fig. 2). The LBC consists of a three axes table. The upper disk can be displaced along the $z$ axis by means of a micrometric screw with an accuracy of $0.01 \mathrm{~mm}$. The lower disk is mounted on a plate which can be displaced parallel to the $x y$ plane by means of two micrometric screws. one of them allowing the displacement of the plate along the $x$ axis and the other along the $y$ axis. Both supporting disks are made of a calibrated stainless steel tube. and the surfaces in contact with the liquid have been carefully polished in order to obtain very sharp edges. During the experiments the disks were kept coaxial, the eccentricity between the centers of the disks being smaller than $0.5 \%$ of the disk radius. Working fluid (distilled water) was injected and removed through a hole in the upper disk connected to a calibrated syringe by flexible tubing.

The LBC, the change-coupled device (CCD) camera, and the illumination system, are mounted on a rotating platform which in tum is mounted on a tilting table. The rotating platform (hence the LBC and the CCD camera) can be rotated as a whole around a fixed point of the tilting table, its position being measured by the rotation angle $\beta$. The LBC is located on the rotation platform in such a way that the liquid bridge axis coincides with the platform rotation axis. The tilting table is articulated along an axis to a fixed base, $\varphi$ being the angle between the tilting table and the fixed base (both angles, $\beta$ and $\varphi$. are measured within $\pm 1^{\circ}$ accuracy). Other pieces of equipment used, but not shown in Fig. 2, are a video recorder, a TV monitor, a PC with an image processor, and the calibrated syringe.

For every experimental run, the experimental procedure has been as follows: First, the tilting table is rotated to the desired nominal value of the tilt angle, $\varphi$, the rotating platform to the nominal value of the rotation angle, $\beta$, and the upper disk is displaced until the desired slendemess, $\Lambda$, is
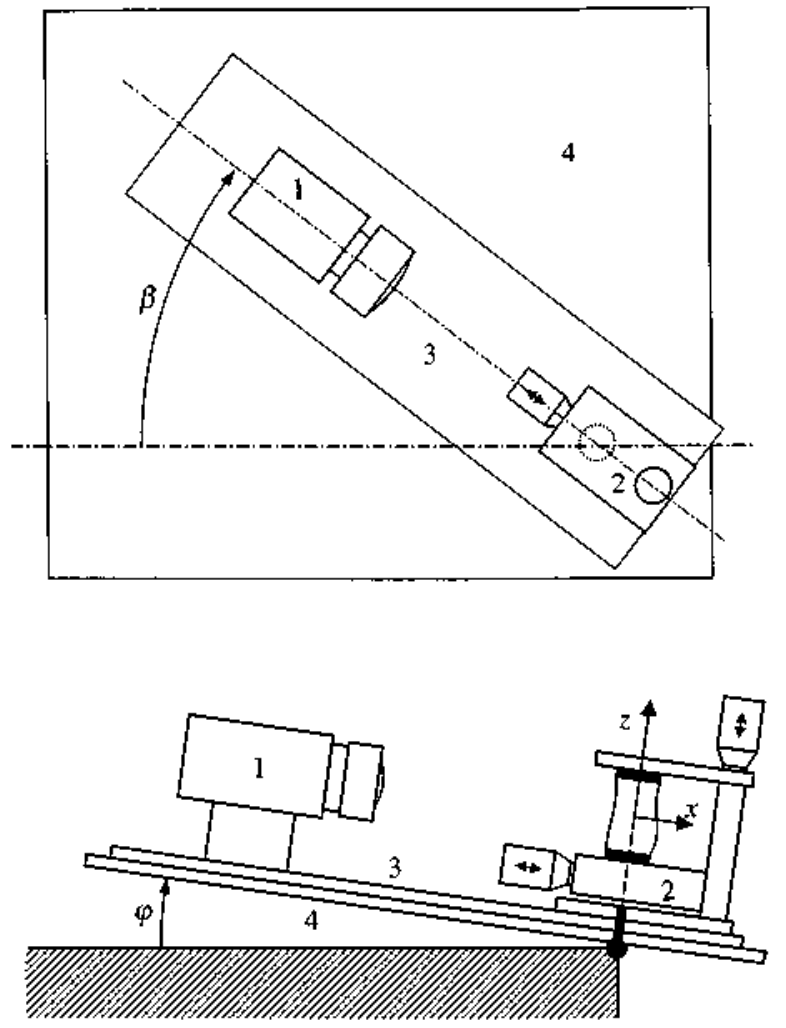

FIG. 2. Experimental arrangenent: (1) CCD canera, (2) nillimetric liquid bridge cell. (3) rotating platform, (4) tilting table.

reached. Then a drop of liquid is injected through the hole in the upper disk and its volume is increased until the drop becomes in contact with the lower disk and a liquid bridge is formed (due to the sharpness of the disk edges the liquid column usually anchors perfectly to the edges of the disks and the spreading of the liquid over the lateral surfaces of the supporting rods during the liquid bridge formation is quite unusual). From this point, the experimental sequence depends on which part of the stability limit curve is being checked. In the case of minimum volume limits, once the liquid bridge is formed the experimental sequence proceeds without any further interaction: Due to water evaporation, the volume of liquid continuously decreases and when the stability limit is reached the liquid column breaks. In the case of maximum volume limits. the volume of liquid is slowly increased by adding water from the syringe until capillary instability develops and the liquid column breakage takes place. In both cases direct manipulation on the LBC is prevented in order to avoid vibrations that could cause the breaking of the liquid column before the corresponding stability limit is reached.

During the experiments, liquid bridge pictures obtained from the $\mathrm{CCD}$ camera are recorded and their contours are digitized every $0.04 \mathrm{~s}$. The last digitized contour before the breaking of the liquid bridge, which is assumed to be the critical shape, is stored for further analysis. The abovedescribed procedure is repeated ten times (to have several experimental data for a given configuration) for every tested value of the rotation angle $\left(\beta=0^{\circ}, 15^{\circ}, 30^{\circ}, 45^{\circ}, 60^{\circ}, 75^{\circ}\right.$, and $90^{\circ}$ ) keeping constant the values of both the slenderness 
and the tilt angle. Then a new value of the slendemess is set and. for every tested value of the tilt angle $\left(\varphi=0^{\circ}, 13^{\circ} .26^{\circ}\right.$. $40^{\circ}, 54^{\circ}, 67^{\circ}, 81^{\circ}$, and $90^{\circ}$ ) the whole process is repeated again until the corresponding stability limit curve is obtained.

For every value of the slenderness and of the tilt angle, the volume of the liquid bridge at the stability limit is computed from the stored critical contours of the liquid column (the last contour before the breaking of the liquid column). According to the experimental procedure. for each value of the tilt angle $\varphi$ and the slenderness $\Lambda$ a set of contours of the critical liquid bridge interface. corresponding to the different values of the rotation angle $\beta$, are obtained. Although each one of the critical contours is obtained in a different experiment, it is assumed that the shape of the liquid bridge at the stability limit, thus the volume of the liquid. is the same no matter the value of the rotation angle. To validate this assumption, and for a representative liquid volume. for each value of the rotation angle the same experiment was repeated many times, and in all cases the digitized contours of the liquid bridge interface were equal, within the experimental error.

In every case the volume of liquid has been calculated using two different procedures: by using the whole set of contours obtained for each values of the tilt angle $\varphi$ and the slenderness $\Lambda$ (details on this procedure can be obtained from the authors upon request) and by using a simplifying assumption considering that the cross sections of the liquid bridge are nearly ellipses. With the latter approximation calculations are much simpler and it suffices to measure the contours corresponding to $\beta=0$ and to $\beta=90^{\circ}$ to obtain just the main axes of the ellipses. Both methods give identical results within experimental accuracy.

To calculate the value of the Bond number during the experiments the procedure was as follows. The tilt angle was set to zero. so that liquid bridges were in vertical position and their interfaces were axisymmetric $\left(\varphi=0, B_{a}=|\mathbf{B}|, B_{l}\right.$ $=0$ ). A liquid bridge with an initial volume higher than the cylindrical one $(r>1)$ is established. The volume of the liquid bridge is continuously computed from the digitized outer shapes while the volume of the liquid column decreases continuously due to evaporation. When the volume is within the range $Y=1 \pm 0.005$ the value of the Bond number is calculated by fitting a second-order accurate theoretical expression for interface shape to that experimentally measured. ${ }^{10}$ The procedure is then repeated for different values of the slenderness and two sets of supporting disks, the results obtained being $B_{a}=|\mathbf{B}|=0.022 \mathrm{I} \pm 0.0002$ for liquid bridges between disks having $0.40 \mathrm{~mm}$ in radius. and $B_{c}$ $=|\mathbf{B}|=0.0232 \pm 0.0002$ in the case of disks with $R$ $=0.41 \mathrm{~mm}$. These values are extremely close to the values of reference which are obtained by taking the nominal values of the plysical magnitudes entering in the definition of the Bond number $\left(\rho=10^{3} \mathrm{~kg} \mathrm{~m}^{-3}, g=9.81 \mathrm{~m} \mathrm{~s}^{-2}\right.$, and $\sigma$ $=0.072 \mathrm{~N} \mathrm{~m}^{-1}$ ), which are $B=0.0218$ and $B=0.0229$, respectively. The small differences can be attributed to the decrease in surface tension due to small contamination. In order to assure that there was not any change in the experimental conditions during the different experimental runs,

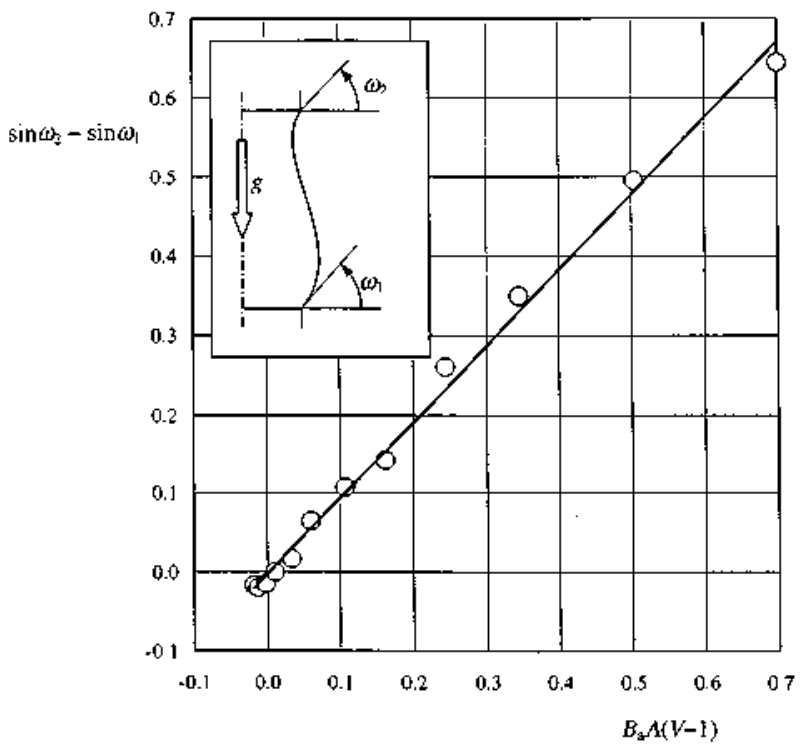

FIG. 3. Variation with the parameter $B_{a} A(Y-1)$ of the difference sin $\alpha_{2}$ $-\sin \omega_{1}$ (where $\omega_{2}$ and $\omega_{1}$ are the critical values of the contact angles at the edges of the bottom and top disks, respectively) of axisymmetric liquid bridges at the stability limit. Symbols indicate experimental results, whereas the solid line is a linear fit to experimental results. According to results obtained by Slobozhanin and Perales (Ref. 14) the slope should be exactly one.

Bond numbers were measured before and after every run and the obtained values were found constant.

\section{EXPERIMENTAL RESULTS}

The first set of experiments was performed with axisymmetric liquid bridges (with the tilting table in the horizontal position, $\varphi=0, B_{a}=|\mathbf{B}|, B_{l}=0$ ) aiming to check some theoretical results predicted by Slobozhanin and Perales. ${ }^{14}$ According to them, the shape of the interface of an axisymmetric liquid bridge at the stability limit (which can be identified by the contact angles at the upper disk, $\omega_{2}$, and at the lower disk, $\omega_{1}$, as indicated in Fig. 3) depends on the Bond number, the slenderness, and the dimensionless volume through the expression: $\sin \omega_{2}-\sin \omega_{1}=B_{a} \Lambda(V-1)$. Experimental results are shown in Fig. 3, where the values of $\sin \omega_{2}$ $-\sin \omega_{1}$ versus the parameter $B_{a g} \Lambda(V-1)$ have been plotted. In this plot the symbols represent experimental results whereas the solid line is a linear least-squares fit to the experimental results. The agreement between the theoretical prediction and the values measured here is excellent.

The stability limits (experimental results) of nonaxisymmetric liquid bridges. critical volume versus slenderness, are shown in Figs. 4(a) and 4(b). Here the stability limit curves corresponding to different values of the tilt angle are plotted. Note that. according to these results, when the volumes are not too large, liquid bridges are more stable when gravity acts along a direction perpendicular to the liquid bridge axis $\left(\varphi=\pi / 2, B_{a}=0, B_{l}=|\mathbf{B}|\right)$ than when it acts in a direction parallel to the liquid bridge axis $\left(\varphi=0, B_{a}=|\mathbf{B}|, B_{l}=0\right)$. This behavior is in complete agreement with theoretical predictions. ${ }^{4.8}$ For instance, the maximum stable slenderness of liquid bridges with slenderness close to the Rayleigh sta- 


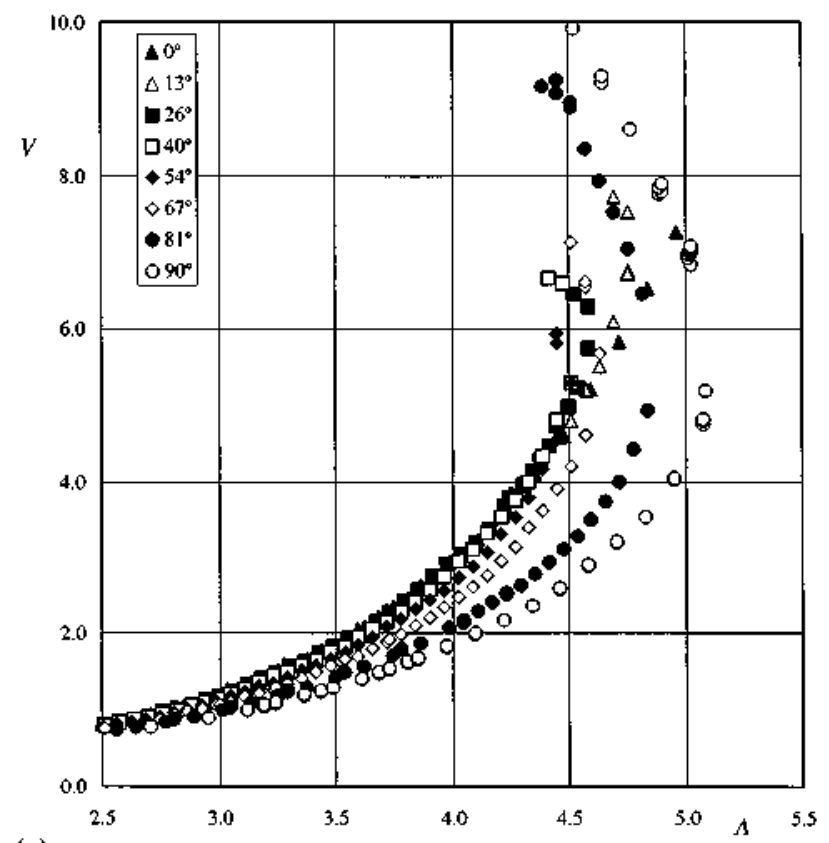

(a)

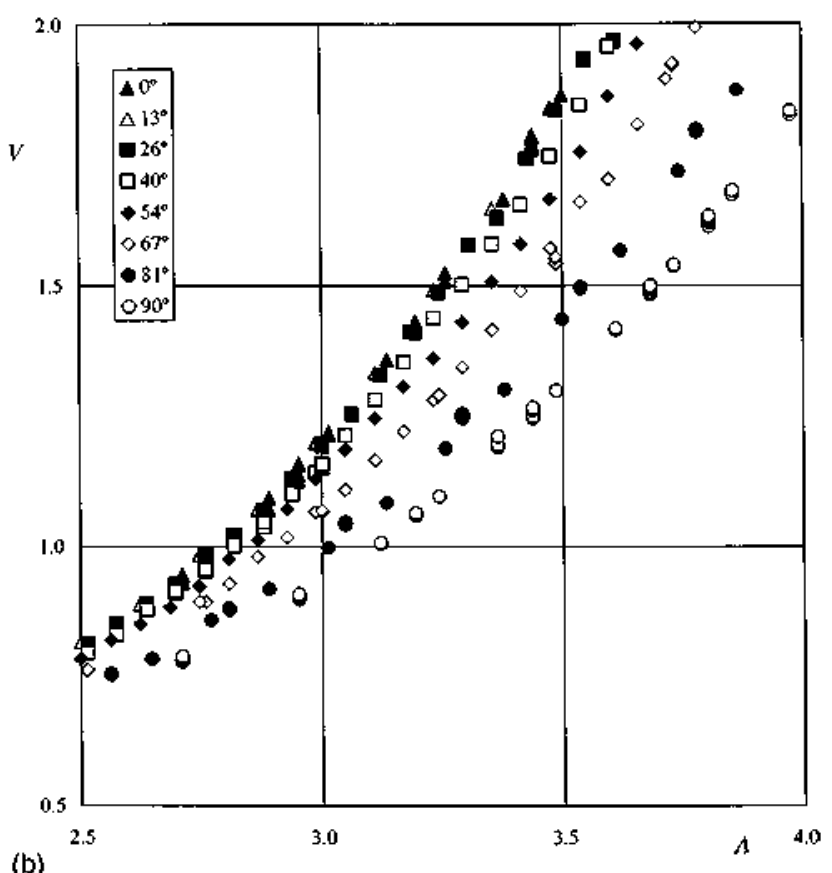

(b)

FIG. 4. Stability limits of liquid bridges between equal, coaxial disks ( $B_{a}$ $=0.0232 \cos \varphi, B_{I}=0.0232 \sin \varphi$ ): (a) whole slenderness range; (b) low slenderness range. The legend indicates the symbols used for different tilt angles, $\varphi$.

bility limit, volume close to that of a cylinder and subjected to very small axial and lateral Bond numbers $(\Lambda \approx \pi, V$ $\approx 1, B_{\mathfrak{a}} \approx 0, B_{l} \approx 0$ ) has been calculated by using standard bifurcation techniques, ${ }^{4}$ the resulting expression for the maximum stable slenderness, $\Lambda_{\text {crit }}$, being

$$
\Lambda_{\text {crit }}=\pi\left[1-\left(\frac{3}{2}\right)^{4 / 3} B_{c i}^{2 i 3}+\frac{1}{2}(V-1)-\frac{\pi^{2}}{4} B_{l}^{2}\right] .
$$

This expression has been plotted in Fig. 5 as well as the values of $\Lambda_{\text {crit }}$ derived from the experimental results shown in Fig. 4(b). According to this plot, the agreement between

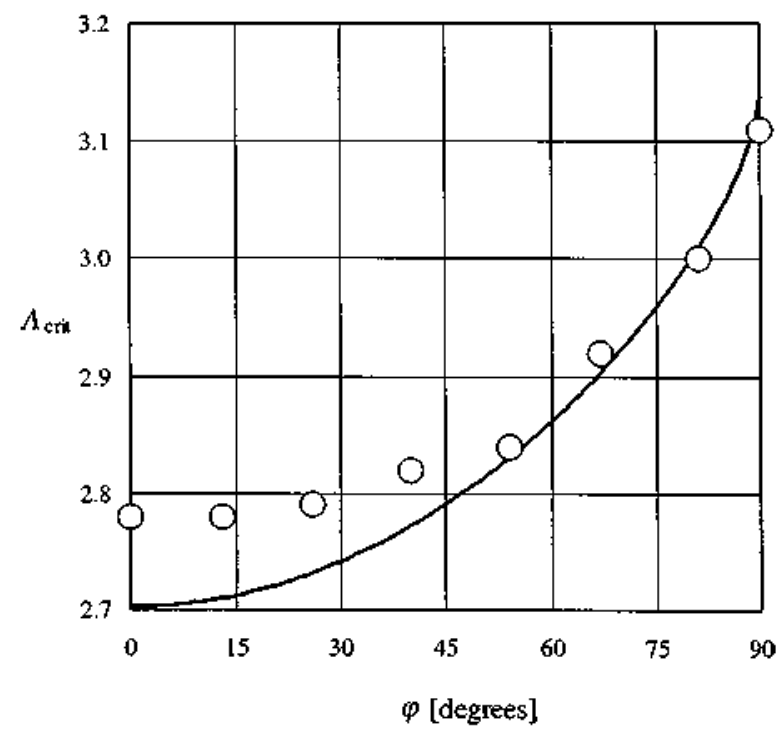

FIG. 5. Variation with the tilt angle, $\varphi$, of the critical length. $\Lambda_{c \mathrm{ml}}$, of liquid bridges with cylindrical volume $(V=1)$. The symbols represent the experimental values, whereas the curve corresponds to the theoretical results according to expression (1).

experimental results and the asymptotic ones increases as the tilt angle grows, as one could expect if the conditions used to deduce expression (1) are recalled

Another remarkable aspect of the experimental results shown in Fig. 4(a) is the existence in each stability limit curve of a maximum slenderness. $\Lambda_{\max }$. that bounds the range of values of the slenderness for which a stable liquid bridge can be formed. As far as we know, the only available results on this topic are the theoretical results published by Slobozhanin and Perales ${ }^{14}$ and Langbein. ${ }^{15}$ and the experi-

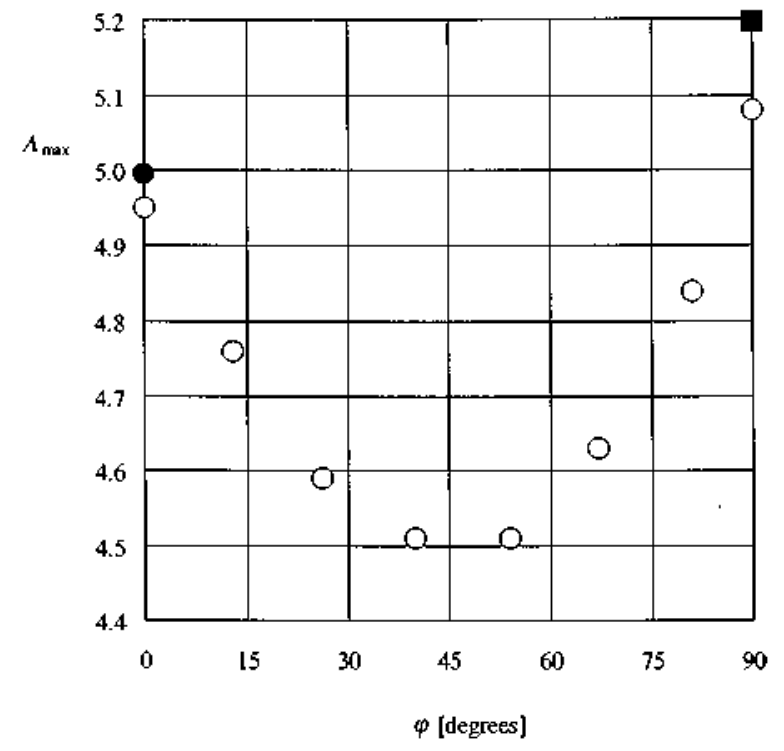

FIG. 6. Variation with the tilt angle, $\varphi$, of the maximum length $\Lambda_{\max }$, of liquid bridges subjected to axial and lateral accelerations $\left(B_{a}\right.$ $\left.=0.0232 \cos \varphi, B_{f}=0.0232 \sin \varphi\right)$. Open symbols represent experimental results whereas the solid ones correspond to the asymptotic result of Langbein-Ref. 15 (circle) and the extrapolated value deduced from the results of Alexander et al.-Ref. 8 (square). 
mental ones published by Bezdenejnykh. Meseguer, and Perales, ${ }^{11}$ although in these cases only axisymmetric liquid bridges were considered ( $\varphi=0, B_{a} \neq 0, B_{l}=0$ ), and the theoretical results of Alexander et $\mathrm{al}^{8}{ }^{8}$ concerning liquid bridges were subjected to either axial or lateral acceleration. The variation of the maximum slenderness with the tilt angle has been represented in Fig. 6 . Note that for the values of the Bond number considered here there is a minimum of $\Lambda_{\max }$ which occurs close to $\varphi=\pi / 4$, that is. when axial and lateral Bond numbers are roughly equal.

\section{ACKNOWLEDGMENT}

This work has been supported by the Spanish Comision Interministerial de Ciencia y Tecnología (CICYT Project No. ESP 95-0029).

'J. Meseguer, L. A. Slobozhanin, and J. M. Perales, "A review on the stability of liquid bridges," Adv. Space Res. 16. 5 (1994).

${ }^{2}$ S. R. Coriell, S. C. Hardy, and M. R. Cordes, "Stability of liquid zones," J. Colloid Interface Sci. 60, 126 (1977)

${ }^{3}$ J. M. Perales, "Non-axisymmetric effects on long liquid bridges." Acta Astron. 15, 561 (1987).

${ }^{4} \mathrm{~J}$. Meseguer, N. A. Bezdenejnykd. J. M. Perales, and P. Rodriguez de Francisco. "Theoretical and experimental analysis of stability limits of nou-axisymmetric licjuid bridges under microgravity conditions," Microgravity Sci. Technol. 8. 2 (1995).

${ }^{5} \mathrm{H}$. Chen and M, Z. Saghir, "Nonaxisymmetric equilibrium shapes of the liquid bridge." Microgravity Sci. Technol. 7, 12 (1994).

${ }^{6}$ A. Laverón-Simavilla and J. M. Perales, "Equilibrium shapes of nonaxisymmetric liquid bridges of arbitrary volume in gravitational fields and their potential energy," Plyys. Fluids 7, 1204 (1995).

${ }^{7}$ A. Laverón-Simavilla and E. Checa, "Effect of a lateral gravitational field on the non-axisymmetric equilibrium shapes of liquid bridges held between eccentric disks and of volumes equal to those of cylinders," Phys. Fluids 9, 817 (1997).

${ }^{8}$ J. I. D. Alexander, S. Delafontaine, A. Resnick, and W. C. Carter, "Stability of non-axisymmetric liquid bridges," Microgravity Sci. Technol. 9. 193 (1996).

${ }^{9}$ M. P. Mahajan, S. Zhang, M. Tsinge, P. L. Taylor, and C. Rosenblatt. "Stability of magnetically levitated liquid bridges of arbitrary volume subjected to axial and lateral gravity," J. Colloid Interface Sci, 123, 592 (1999).

${ }^{10} \mathrm{~J}$. Meseguer, N. A. Bezdenejinykh, and P. Rodriguez de Francisco, "On the use of liquid bridges as accelerometers," Microgravity Sci. Technol. 9. 62 (1996).

"N. A. Bezdenejnykh, J, Meseguer, and J. M. Perales, "Experimental analysis of stability limits of capillary liquid bridges," Phys. Flujds A 4. 677 (1992)

${ }^{12}$ A. Resuick and J. I. D. Alexander, "Plateau tank apparatus for the study of licjuid bridges," Rev. Sci. Instrum. 68, 1495 (1997).

${ }^{13} \mathrm{H}$. González and A. Castellanos, "The effect of residual gravity on the stability of liquid colunns subjected to electric fields," J. Fluid Mech. 249. 185 (1993).

${ }^{14}$ L. A. Slobozhanin and J. M. Perales. "Stability of liquid bridges between equal disks in an axial gravity field,"' Phys. Fluids A 5, 1305 (1993).

${ }^{15}$ D. Langhein, "Crystal growth from liquid colunus,", J. Cryst. Growth 104. 47 (1990). 